\title{
Testing of Model Variant
}

National Cancer Institute

\section{Source}

National Cancer Institute. Testing of Model Variant. NCI Thesaurus. Code C139450.

The investigation employed relevant empirical testing of a model variant of the device involved in the reported adverse event in order to support the identification of possible causes for the adverse event through plausibility reasoning. A model variant is not identical to the actual device, but shares relevant characteristics with the device involved. Relevant testing would typically be based on test methods used for evaluating safety and performance as described in the latest relevant standards. 